\title{
SYNTHESIS OF CERTAIN 2-AROYLINDOLE DERIVATIVES OF POTENTIAL ANALGESIC, ANTI-INFLAMMATORY AND ANTIPYRETIC ACTIVITIES
}

\author{
S. G. Abdel-Moty*, A. M. Abdel-Aal, A. N. Kafafy and A. A. El-Shorbagi \\ Department of Pharmaceutical Organic Chemistry, Faculty of Pharmacy, Assiut University, \\ Assiut 71526, Egypt
}

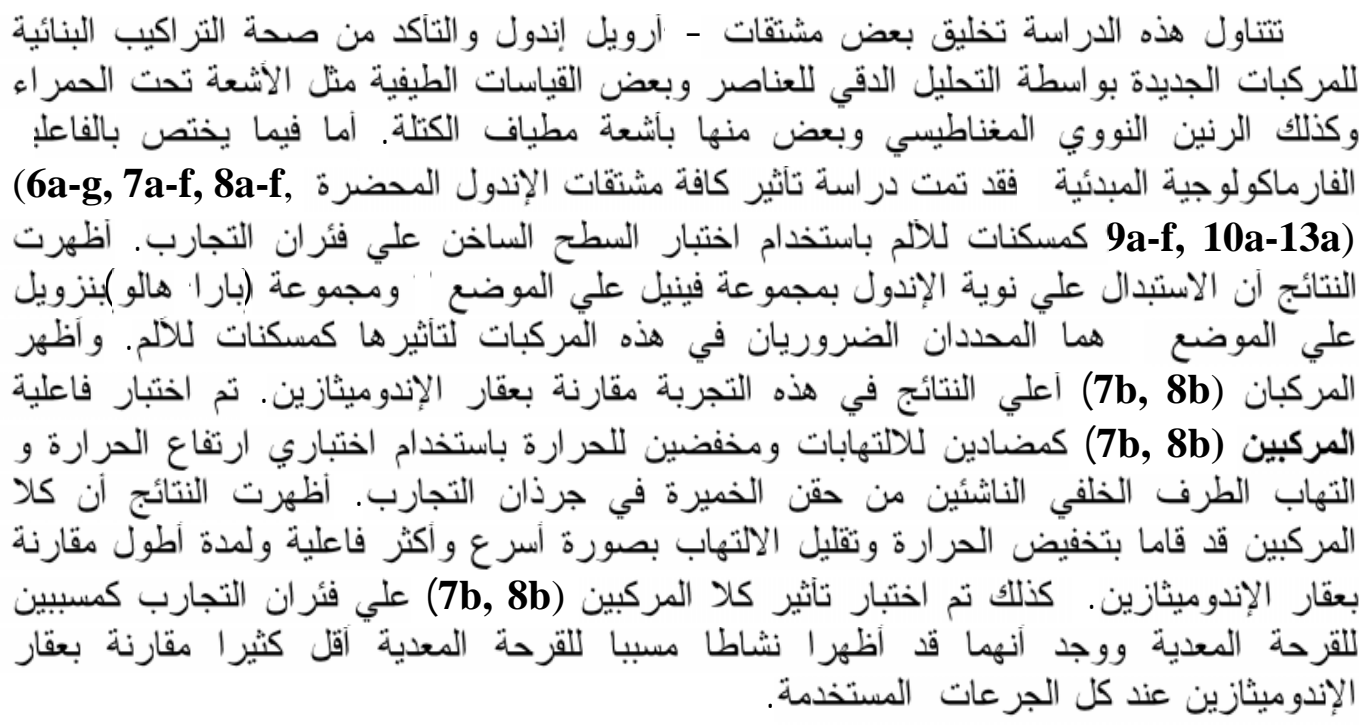

In the present study, a series of 2-aroylindole derivatives were synthesized by phase transfer catalysis (PTC) and were characterized by $I R,{ }^{1} H-N M R$, Mass spectral and Elemental analysis. Indole derivatives $\mathbf{6 a - g}, 7 a-f, 8 a-f$ and $9 a-13 a$ were tested for analgesic activity using hot-plate test. Compounds $7 b$ and $8 b$ were tested for antipyretic and anti-inflammatory activity using yeast induced hyperthermia and paw edema in rats. Analgesic activity was shown when indole nucleus was substituted at position 2 and 3 by phenyl and (p-halo)benzoyl moieties respectively, where highest activity was recognised in compounds $\mathbf{7 b}$ and $8 \boldsymbol{b}$. Both compounds also exhibited faster, more effective and prolonged reduction in hyperthermia and edema induced in rats compared with indomethacin. Compounds $\mathbf{7 b}$ and $8 \boldsymbol{b}$ were also tested for ulcerogenic activity in mice, where a lower ulcerogenic effect was observed compared with indomethacin at all tested dose levels.

\section{INTRODUCTION}

Synthesis, reactivity and wide range of biological activities found among indoles have been a topic of research interest. Great interest has been focused on indomethacin as a drug used in the treatment of rheumatoid arthritis and related conditions. Modification of its structure, specifically, at nitrogen or variations of attachment position of the benzoyl moiety at position 2 or 3 of the indole nucleus led to anti- inflammatory and analgesic compounds. ${ }^{1-10}$ In addition, the anti-inflammatory potencies of zomepirac, tolmetine and ketorolac are wellknown. This class of compounds is characterized by an aryl-heteroaryl ketone arrangement. ${ }^{11-14}$

Based on the above-mentioned findings, it seemed reasonable to synthesize a series of 2aroylindoles that incorporate the methanone moiety linking the aryl and the heteroaryl (indole) nucleus. Selection of the different 
substituents was based on the following rationale:

a) Simple alkyl groups of increasing carbon chain (e.g. methyl, ethyl, n-propyl, n-butyl) would find out whether $\mathrm{N}$-alkylation and/or chain lengthening has any effect on biological response.

b) The $p$-substituent $\left(\mathrm{H}, \mathrm{Cl}, \mathrm{Br}, \mathrm{CH}_{3}, \mathrm{NO}_{2}\right)$ of the benzoyl entity would evaluate the $p$ substituent effect. These substituents were capable to correlate the physico-chemical parameters with the anticipated biological activity, if any.

c) The $N$-benzyl moiety would explore the effect of introducing an aryl entity separated by one carbon from the nitrogen atom of the indole ring.

d) The $N$-benzoyl group was chosen to be of closer structural approach to indomethacin.

e) Substitution at position 3 of the indole nucleus by methyl or phenyl was chosen to explore the importance of existence of arylaroyl arrangement for biological response.

\section{Chemistry}

Synthesis of 1-alkyl-2-aroylindoles (6b-f, $\mathbf{7 b - f}, \mathbf{8 b - f}$ and $\mathbf{9 b - f}$ ) was attempted by two different approaches. The first one was through cyclo-condensation of $\mathrm{N}$-alkyl-o-aminobenzophenone (1),c) with appropriate phenacyl bromide (5a,d), leading to compounds $\mathbf{6 c}$ and 9b, respectively, Fig 1 . The second approach involved $\mathrm{N}$-alkylation of the already cyclized 2 aroylindoles (6a-13a) using solid-liquid phase transfer catalysis (SL-PTC); resulting in compounds $\{$ 6-9 (b-f) $\}$, Fig. 1.

2-Aroylindoles (6a-13a) in their turn were obtained by treating the appropriate $o$ aminophenyl ketones with suitable phenacyl bromide, Fig.1. Moreover, synthesis of 1,2Dibenzoyl-3-phenylindole (6g) was achieved through base-catalyzed acylation of 2-benzoyl3-phenylindole (6a), Fig. 1.

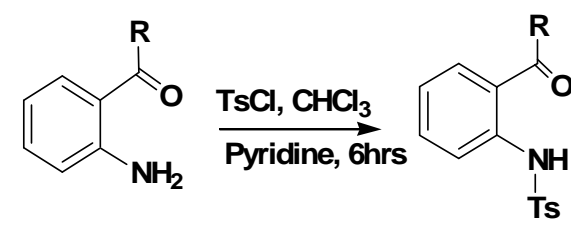

1a, 2a
$3 a, 4 a$

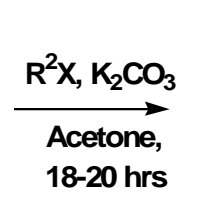

$3 b \& c, 4 b \& c$

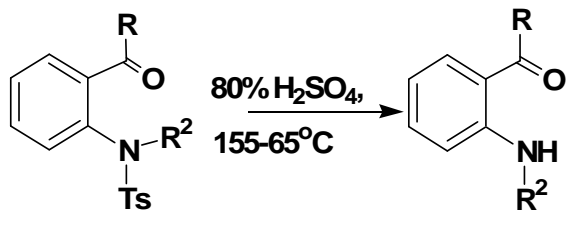

$1 b \& c, 2 b \& c$

(1b-c)

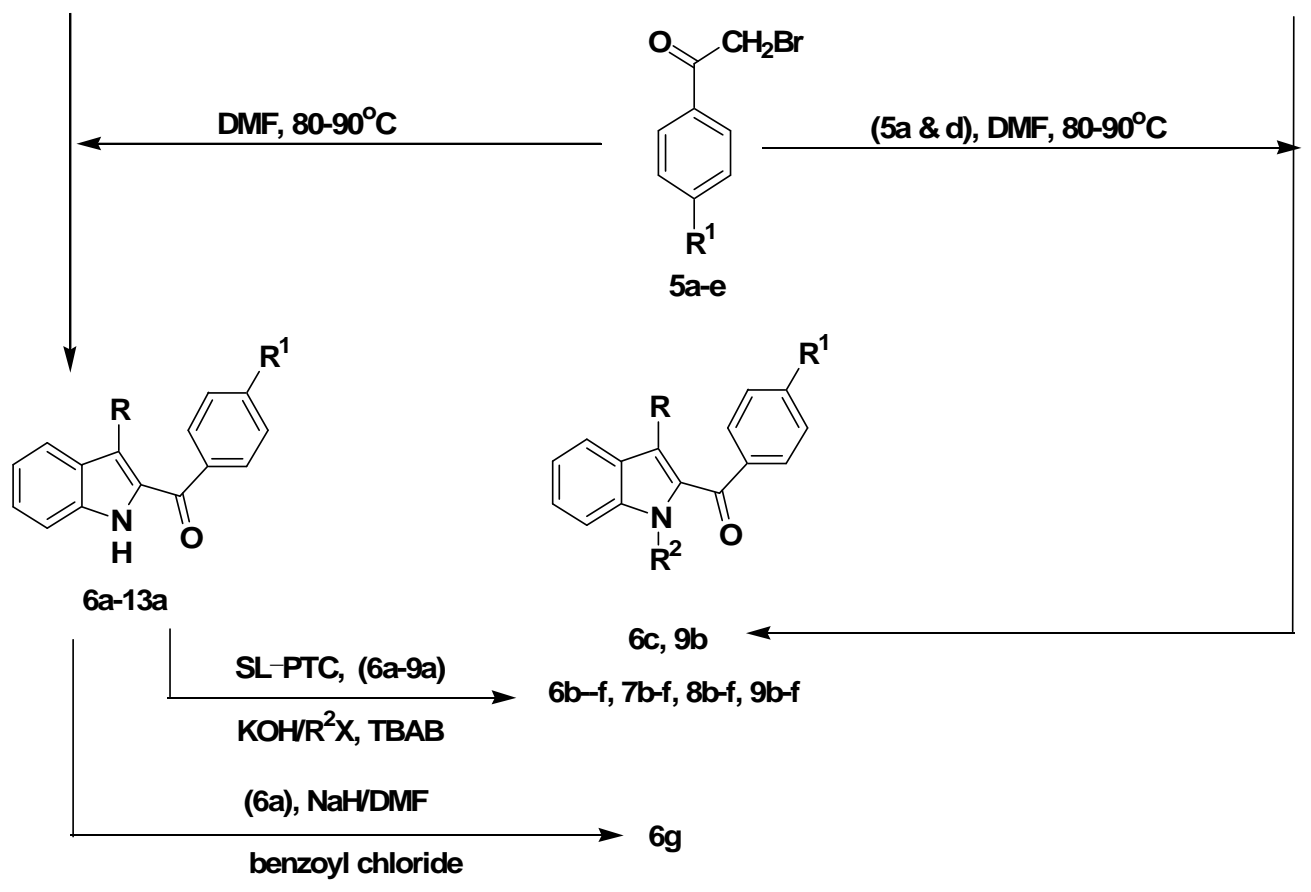

Fig. 1: Synthesis of 1-alkyl-2-aroylindoles (first approach) \& N-alkylation and N-acylation of 2-aroylindoles (second approach). 


\section{Pharmacology}

The effect of synthesized compounds on mean licking time in hot-plate test was used as a measure of analgesic activity. Also, antipyretic activity of compounds $\mathbf{7 b}$ and $\mathbf{8 b}$ was investigated using yeast induced hyperthermia test. In addition, both the time course of edema and percentage inhibition of edema were used as measures for antiinflammatory activity of compounds $\mathbf{7 b}$ and $\mathbf{8 b}$ using yeast induced edema. Activities of the tested compounds were compared with indomethacin as a reference drug at 5 and 10 $\mathrm{mg} / \mathrm{kg}$ dose levels. Compounds $\mathbf{7 b}$ and $\mathbf{8 b}$ were then tested for ulcerogenic activity in mice.

\section{EXPERIMENTAL}

\section{Chemistry}

M.p were determined in open capillary tubes and are uncorrected. Precoated silica gel plates (kieselgel 60 GF, Merck, Germany) were used for TLC. IR spectra were recorded (in $\mathrm{KBr}$ ) on a Shimadzu 200 spectrophotometer. ${ }^{1} \mathrm{H}-\mathrm{NMR}$ spectra were recorded on Varian EM$36060 \mathrm{MHz}$ NMR Spectrometer using $\mathrm{CDCl}_{3}$ as a solvent and were reported as $\delta$ values in ppm relative to tetramethylsilane (TMS) as internal standard. Elemental analysis was performed on Elementar Analysenysteme $\mathrm{GmbH}$ VarioEL V2.3 07. Analyses indicated by the symbols of the elements are within $\pm 0.4 \%$ of the theoretical values. Mass spectra were performed on a JOEL JMS600 spectrometer.

\section{General procedure for N-tosyl- o- aminophenyl ketones $3 a$ and $4 a^{15}$}

Tosyl chloride $(0.1 \mathrm{~mol})$ dissolved in chloroform $(50 \mathrm{ml})$ was added dropwise to a stirred solution of $o$-aminophenyl ketone (1a, 2a; $0.1 \mathrm{~mol})$ and pyridine $(0.1 \mathrm{~mol})$ in chloroform $(250 \mathrm{ml})$ at $0^{\circ}$. The reaction mixture was stirred for $6 \mathrm{hrs}$ then washed with water $(2 \times 40 \mathrm{ml}), \mathrm{HCl}(2 \times 30 \mathrm{ml}, 10 \%)$ and water $(2 \times 40 \mathrm{ml})$. The organic layer was separated, dried using anhydrous sodium sulfate and evaporated under vacuum leaving a solid which was crystallized from ethanol.

N-Tosyl-o-aminobenzophenone (3a) Yield $85 \%$, m.p 127-129 . IR $\left(\mathrm{KBr}, \mathrm{cm}^{-1}\right): 3250(\mathrm{~N}-$ $\mathrm{H}), 1644(\mathrm{C}=\mathrm{O}), 1394,1184\left(\mathrm{SO}_{2}\right) .{ }^{1} \mathrm{H}-\mathrm{NMR}$ $\left(\mathrm{CDCl}_{3}, \delta, \mathrm{ppm}\right): 2.2\left(\mathrm{~s}, 3 \mathrm{H}\right.$, tolyl $\left.\mathrm{CH}_{3}\right), 6.9-7.9$ (m, $13 \mathrm{H}$, tolyl $\mathrm{C}_{6} \mathrm{H}_{4}, \mathrm{C}_{6} \mathrm{H}_{5}$ and $o$-disubstituted $\mathrm{C}_{6} \mathrm{H}_{4}$ ), 10.0 (s, $1 \mathrm{H}, \mathrm{NH} ; \mathrm{D}_{2}$ Oexchange). Anal. Calc. for $\mathrm{C}_{20} \mathrm{H}_{17} \mathrm{NO}_{3} \mathrm{~S}$ : C, 68.36; H, 4.88; N, 3.99; S, 9.12. Found: C, 68.34; H, 4.66; N, 3.96; S, $9.0 \%$.

N-Tosyl-o-aminoacetophenone (4a) Yield: $82 \%$, m.p 154-157 ${ }^{\circ} \mathrm{C}$. IR $\left(\mathrm{KBr}, \mathrm{cm}^{-1}\right): 3350(\mathrm{~N}-$ $\mathrm{H}), 1646(\mathrm{C}=\mathrm{O}), 1338,1164\left(\mathrm{SO}_{2}\right) .{ }^{1} \mathrm{H}-\mathrm{NMR}$ $\left(\mathrm{CDCl}_{3}, \delta, \mathrm{ppm}\right): 2.4\left(\mathrm{~s}, 3 \mathrm{H}\right.$, tolyl $\left.\mathrm{CH}_{3}\right), 2.5$ (s, $\left.3 \mathrm{H}, \mathrm{COCH}_{3}\right), 7.2-7.5\left(\mathrm{~m}, 8 \mathrm{H}\right.$, tolyl $\mathrm{C}_{6} \mathrm{H}_{4}$ and odisubstituted $\left.\mathrm{C}_{6} \mathrm{H}_{4}\right), \quad 11.5 \quad(\mathrm{~s}, \quad 1 \mathrm{H}, \quad \mathrm{NH}$; $\mathrm{D}_{2}$ Oexchange). Anal. Calc. for $\mathrm{C}_{15} \mathrm{H}_{15} \mathrm{NO}_{3} \mathrm{~S}$ : C, 62.26; H, 5.23; N, 4.84; S, 11.08. Found: C, 62.17 ; H, 5.38; N, 4.86; S, $10.81 \%$.

\section{General procedure for N-alkyl-N-tosyl-o- aminophenyl ketones 3 and $4(\mathrm{~b}, \mathrm{c})^{15}$}

The appropriate alkyl halide $(0.02 \mathrm{~mol})$ was added to a suspension of $N$-tosyl-oaminophenyl ketone 3a, 4a $(0.01 \mathrm{~mol})$ and potassium carbonate $(0.02 \mathrm{~mol})$ in acetone (30 $\mathrm{ml})$. The reaction was stirred for 18-20 hrs at r.t. The suspension was evaporated under vacuum and the residue was extracted with chloroform $(5 \times 30 \quad \mathrm{ml})$. The combined chloroform extracts was washed with water $(3 \times 20 \mathrm{ml})$, dried with anhydrous sodium sulfate and evaporated under vacuum. The residue was crystallized from ethanol.

N-Methyl-N-tosyl-o-aminobenzophenone (3b) Yield: $84 \%$, m.p 126-128. $\operatorname{IR}\left(\mathrm{KBr}, \mathrm{cm}^{-1}\right)$ : $1665(\mathrm{C}=\mathrm{O}), \quad 1337,1157 \quad\left(\mathrm{SO}_{2}\right) .{ }^{1} \mathrm{H}-\mathrm{NMR}$ $\left(\mathrm{CDCl}_{3}, \delta, \mathrm{ppm}\right): 2.4\left(\mathrm{~s}, 3 \mathrm{H}\right.$, tolyl $\left.\mathrm{CH}_{3}\right), 3.1(\mathrm{~s}$, $\left.3 \mathrm{H}, \mathrm{NCH}_{3}\right)$, 6.8-7.9 (m, 13H, tolyl $\mathrm{C}_{6} \mathrm{H}_{4}, \mathrm{C}_{6} \mathrm{H}_{5}$ and o-disubstituted $\mathrm{C}_{6} \mathrm{H}_{4}$ ). Anal. Calc. for $\mathrm{C}_{21} \mathrm{H}_{19} \mathrm{NO}_{3} \mathrm{~S}: \mathrm{C}, 69.02 ; \mathrm{H}, 5.24 ; \mathrm{N}, 3.83 ; \mathrm{S}$, 8.77. Found: C, 68.63; H, 5.16; N, 3.79; S, $8.58 \%$.

N-Methyl-N-tosyl-o-aminoacetophenone (4b) Yield: 82\%, m.p 116-118 . IR (KBr, cm $\left.{ }^{1}\right)$ : $1685(\mathrm{C}=\mathrm{O}), 1339,1170 .\left(\mathrm{SO}_{2}\right) \cdot{ }^{1} \mathrm{H}-\mathrm{NMR}$ $\left(\mathrm{CDCl}_{3}, \delta, \mathrm{ppm}\right): 2.4\left(\mathrm{~s}, 3 \mathrm{H}\right.$, tolyl $\left.\mathrm{CH}_{3}\right), 2.7(\mathrm{~s}$, $\left.3 \mathrm{H}, \mathrm{COCH}_{3}\right), 3.3\left(\mathrm{~s}, 3 \mathrm{H}, \mathrm{NCH}_{3}\right), 6.7-7.4(\mathrm{~m}$, $8 \mathrm{H}$, tolyl $\mathrm{C}_{6} \mathrm{H}_{4}$ and $o$-disubstituted $\mathrm{C}_{6} \mathrm{H}_{4}$ ). Anal. Calc. for $\mathrm{C}_{16} \mathrm{H}_{17} \mathrm{NO}_{3} \mathrm{~S}: \mathrm{C}, 63.34 ; \mathrm{H}, 5.65 ; \mathrm{N}$, 4.62; S, 10.57. Found: C, 63.41; H, 5.39; N, $4.61 ; \mathrm{S}, 10.45 \%$.

N-Ethyl-N-tosyl-o-aminobenzophenone

(3c) Yield: $91 \%$, m.p 102-104 ${ }^{\circ}$ IR $\left(\mathrm{KBr}, \mathrm{cm}^{-1}\right)$ : $1662(\mathrm{C}=\mathrm{O}), \quad 1339,1169 \quad\left(\mathrm{SO}_{2}\right) .{ }^{1} \mathrm{H}-\mathrm{NMR}$ $\left(\mathrm{CDCl}_{3}, \delta, \mathrm{ppm}\right): 1.2\left(\mathrm{t}, 3 \mathrm{H}, \mathrm{NCH}_{2} \mathrm{CH}_{3}\right), 2.3(\mathrm{~s}$, $3 \mathrm{H}$, tolyl $\mathrm{CH}_{3}$ ), 3.7 (q, $2 \mathrm{H}, \mathrm{NCH}_{2} \mathrm{CH}_{3}$ ), 7-7.9 $\left(\mathrm{m}, 13 \mathrm{H}\right.$, tolyl $\mathrm{C}_{6} \mathrm{H}_{4}, \mathrm{C}_{6} \mathrm{H}_{5}$ and $o$-disubstituted 
$\mathrm{C}_{6} \mathrm{H}_{4}$ ). Anal. Calc. for $\mathrm{C}_{22} \mathrm{H}_{21} \mathrm{NO}_{3} \mathrm{~S}$ : C, 69.63; H, 5.58; N, 3.69; S, 8.45. Found: C, 69.42; H, $6.00 ; \mathrm{N}, 3.66 ; \mathrm{S}, 8.33 \%$.

$\mathrm{N}$-Ethyl-N-tosyl-o-aminoacetophenone (4c) Yield: $96 \%$, m.p $136-138{ }^{\circ} \mathrm{C}$. IR $\left(\mathrm{KBr}, \mathrm{cm}^{-}\right.$ $\left.{ }^{1}\right)$ : $1696(\mathrm{C}=\mathrm{O}), 1338,1169\left(\mathrm{SO}_{2}\right) .{ }^{1} \mathrm{H}-\mathrm{NMR}$ $\left(\mathrm{CDCl}_{3}, \delta, \mathrm{ppm}\right): 1.2\left(\mathrm{t}, 3 \mathrm{H}, \mathrm{NCH}_{2} \mathrm{CH}_{3}\right), 2.5(\mathrm{~s}$, $3 \mathrm{H}$, tolyl $\left.\mathrm{CH}_{3}\right), 2.7\left(\mathrm{~s}, 3 \mathrm{H}, \mathrm{COCH}_{3}\right), 3.7$ (q, $2 \mathrm{H}$, $\left.\mathrm{NCH}_{2} \mathrm{CH}_{3}\right), 6.8-7.5\left(\mathrm{~m}, 8 \mathrm{H}\right.$, tolyl $\mathrm{C}_{6} \mathrm{H}_{4}$ and $o-$ disubstituted $\mathrm{C}_{6} \mathrm{H}_{4}$ ). Anal. Calc. for $\mathrm{C}_{17} \mathrm{H}_{19} \mathrm{NO}_{3} \mathrm{~S}: \mathrm{C}, 64.33 ; \mathrm{H}, 6.03 ; \mathrm{N}, 4.41 ; \mathrm{S}$, 10.10. Found: C, 64.23; H, 5.78; N, 4.41; S, $10.20 \%$.

\section{Detosylation reaction- General procedure for preparation of $\mathrm{N}$ - alkyl- o-aminophenyl ketones 1 and 2 (b,c)}

To N-alkyl-N-tosyl-o-aminophenyl ketone 3 and 4 (b, c); (0.01 mol) was added sulfuric acid $(5 \mathrm{ml}, 80 \%)$. The mixture was heated with stirring at $155-65^{\circ}$ and stirred for about $5 \mathrm{~min}$ until the solid passed into solution. The solution was left to cool and poured onto water (25-30 ml). The solution was rendered alkaline using a solution of sodium hydroxide (20\%) and left overnight in refrigerator. The residue obtained was either crystallized from ethanol $(\mathbf{1 b}, \mathbf{c})$ or separated using dry column flash chromatography $(\mathbf{2 b}, \mathbf{c})$ where the elution system was hexane: ethyl acetate (9:1).

N-Methyl-o-aminobenzoophenone Yield: $80 \%$, m.p $70-72^{\circ}$. IR $\left(\mathrm{KBr}, \mathrm{cm}^{-1}\right): 3355$ $(\mathrm{N}-\mathrm{H}), 1618(\mathrm{C}=\mathrm{O}), 740,697$ (bending of mono substituted $\mathrm{Ar}-\mathrm{H}) .{ }^{1} \mathrm{H}-\mathrm{NMR}\left(\mathrm{CDCl}_{3}, \delta\right.$, ppm): 3.0 (d, $3 \mathrm{H}, \mathrm{NCH}_{3}$ ), 6.5-7.8 (m, $9 \mathrm{H}, \mathrm{C}_{6} \mathrm{H}_{5}$ and $o$-disubstituted $\mathrm{C}_{6} \mathrm{H}_{4}$ ), 8.6 (hump, $1 \mathrm{H}, \mathrm{NH}$; $\mathrm{D}_{2}$ Oexchange). Anal. Calc. for $\mathrm{C}_{14} \mathrm{H}_{13} \mathrm{NO}$ : $\mathrm{C}$, 79.59; H, 6.20; N, 6.63. Found: C, 78.95; H, $6.26 ; \mathrm{N}, 6.54 \%$.

N-Methyl-o-aminoacetophenone

Yield: $82 \%$, oil. IR $\left(\mathrm{KBr}, \mathrm{cm}^{-1}\right): 3315(\mathrm{~N}-\mathrm{H})$, $1634(\mathrm{C}=\mathrm{O}) .{ }^{1} \mathrm{H}-\mathrm{NMR}\left(\mathrm{CDCl}_{3}, \delta, \mathrm{ppm}\right): 2.6(\mathrm{~s}$, $\left.3 \mathrm{H}, \mathrm{COCH}_{3}\right), 2.9\left(\mathrm{~d}, 3 \mathrm{H}, \mathrm{NCH}_{3}\right), 6.7-7.7(\mathrm{~m}$, $4 \mathrm{H}, o$-disubstituted $\mathrm{C}_{6} \mathrm{H}_{4}$ ), 8.8 (hump, $1 \mathrm{H}, \mathrm{NH}$; $\mathrm{D}_{2}$ Oexchange).

N-Ethyl-o-aminobenzoophenone Yield: $81 \%$, m.p $43-45^{\circ}$. IR $\left(\mathrm{KBr}, \mathrm{cm}^{-1}\right)$ : 3335 $(\mathrm{N}-\mathrm{H}), 1617(\mathrm{C}=\mathrm{O})$, 750, $702(\mathrm{Ar}-\mathrm{H}) .{ }^{1} \mathrm{H}-\mathrm{NMR}$ $\left(\mathrm{CDCl}_{3}, \delta, \mathrm{ppm}\right): 1.3\left(\mathrm{t}, 3 \mathrm{H}, \mathrm{NCH}_{2} \mathrm{CH}_{3}\right), 3.2$ (m, 2H, $\mathrm{NCH}_{2} \mathrm{CH}_{3}$ ), 6.3-6.8; 7.2-7.7 (m, 9H, $\mathrm{C}_{6} \mathrm{H}_{5}$ and $o$-disubstituted $\mathrm{C}_{6} \mathrm{H}_{4}$ ), 8.5 (hump, $1 \mathrm{H}$, $\mathrm{NH} ; \mathrm{D}_{2}$ Oexchange).
N-Ethyl-o-aminoacetophenone (2c) Yield: 82\%, oil. IR $\left(\mathrm{KBr}, \mathrm{cm}^{-1}\right): 3315(\mathrm{~N}-\mathrm{H}), 1633$ $(\mathrm{C}=\mathrm{O}) .{ }^{1} \mathrm{H}-\mathrm{NMR}\left(\mathrm{CDCl}_{3}, \delta, \mathrm{ppm}\right): 1.3(\mathrm{t}, 3 \mathrm{H}$, $\left.\mathrm{NCH}_{2} \mathrm{CH}_{3}\right), 2.6\left(\mathrm{~s}, 3 \mathrm{H}, \mathrm{COCH}_{3}\right), 3.2(\mathrm{~m}, 2 \mathrm{H}$, $\left.\mathrm{NCH}_{2} \mathrm{CH}_{3}\right), \quad 6.7-7.5 ; \quad 7.2-7.7 \quad(\mathrm{~m}, \quad 4 \mathrm{H}, \quad o-$ disubstituted $\mathrm{C}_{6} \mathrm{H}_{4}$ ), 8.8 (hump, $1 \mathrm{H}, \mathrm{NH}$; $\mathrm{D}_{2}$ Oexchange).

General procedure for preparation of 2substituted benzoyl-3- methyl/ phenylindoles 6-13 (a)

The appropriate $o$-aminophenylketone $(\mathbf{1}$, 2 (a), $0.02 \mathrm{~mol}$ ) and the appropriate phenacyl bromide (5a-e, $0.02 \mathrm{~mol}$ ) were dissolved in anhydrous DMF $(50 \mathrm{ml})$. The solution was heated in an oil bath $\left(80-90^{\circ}\right)$ for 12-16 hrs, monitored by TLC [hexane: ethyl acetate $(9: 1)]$. The reaction mixture was then poured on ice-water. The residue was filtered, dried and crystallized from methanol.

2-Benzoyl-3-phenyl-1H-indole (6a) Yield: $73 \%$, m.p 203-204 ${ }^{\circ}$ as reported. ${ }^{16}$

2-(4-Bromobenzoyl)-3-phenyl-1H-indole (7a) Yield: $60 \%$, m.p 188-189. $\mathrm{IR}\left(\mathrm{KBr}, \mathrm{cm}^{-1}\right)$ : $3330(\mathrm{~N}-\mathrm{H}), 1610(\mathrm{C}=\mathrm{O}), 763,712(\mathrm{Ar}-\mathrm{H}) .{ }^{1} \mathrm{H}-$ NMR $\left(\mathrm{CDCl}_{3}, \delta, \mathrm{ppm}\right): 7.1-7.9\left(\mathrm{~m}, 13 \mathrm{H}, \mathrm{C}_{6} \mathrm{H}_{5}\right.$, $\mathrm{COC}_{6} \mathrm{H}_{4} \mathrm{Br}, o$-disubstituted $\mathrm{C}_{6} \mathrm{H}_{4}$ ), 9.5 (hump, $1 \mathrm{H}, \mathrm{NH})$. Anal. Calc. for $\mathrm{C}_{21} \mathrm{H}_{14} \mathrm{BrNO}$ : C, 67.04; H, 3.75; N, 3..72. Found: C, 67.04; H, $3.34 ; \mathrm{N}, 3.72 \%$. MS (FAB+), m/z: 376.98 $(0.5 \%, \mathrm{M}+\mathrm{H}), 378.92(0.3 \%, \mathrm{M}+\mathrm{H}+2)$.

2-(4-Chlorobenzoyl)-3-phenyl-1H-indole (8a) Yield: 55\%, m.p 179-180 ${ }^{\circ}$ as reported. ${ }^{17}$

2-(4-Methylbenzoyl)-3-phenyl-1H-indole

(9a) Yield: $83 \%$, m.p 156-158 (reported m.p $148-149^{\circ} .^{17}$

2-(4-Nitrobenzoyl)-3-phenyl-1H-indole (10a) Yield: $40 \%$, m.p $182-184^{\circ}$. IR (KBr, cm $\left.{ }^{1}\right)$ : $3340(\mathrm{~N}-\mathrm{H}), 1613(\mathrm{C}=\mathrm{O}), 770,713(\mathrm{Ar}-\mathrm{H})$. ${ }^{1} \mathrm{H}-\mathrm{NMR}\left(\mathrm{CDCl}_{3}, \delta, \mathrm{ppm}\right): 7.1-8.1(\mathrm{~m}, 13 \mathrm{H}$, $\mathrm{C}_{6} \mathrm{H}_{5}, \mathrm{COC}_{6} \mathrm{H}_{4} \mathrm{NO}_{2}$, o-disubstituted $\left.\mathrm{C}_{6} \mathrm{H}_{4}\right), 9.5$ (hump, $1 \mathrm{H}, \mathrm{NH} ; \mathrm{D}_{2}$ Oexchange). Anal. Calc. for $\mathrm{C}_{21} \mathrm{H}_{14} \mathrm{~N}_{2} \mathrm{O}_{3}$ : C, 73.68; $\mathrm{H}, 4.12 ; \mathrm{N}, 8.18$. Found: C, 73.10; H, 3.91; N, 8.11\%.

2-Benzoyl-3-methyl-1H-indole(11a)Yield: $35 \%$, m.p $138-140^{\circ}$ as reported. ${ }^{16}$

2-(4-Bromobenzoyl)-3-methyl-1H-indole (12a) Yield: $25 \%$, m.p 182-184 ${ }^{\circ}$ IR $(\mathrm{KBr}, \mathrm{cm}$ 1): $3320(\mathrm{~N}-\mathrm{H}), 1611(\mathrm{C}=\mathrm{O}), 835,740(\mathrm{Ar}-\mathrm{H})$. ${ }^{1} \mathrm{H}-\mathrm{NMR}\left(\mathrm{CDCl}_{3}, \delta, \mathrm{ppm}\right): 2.3$ (s, 3H, $\left.\mathrm{CH}_{3}\right)$, 7$7.8\left(\mathrm{~m}, 8 \mathrm{H}, \mathrm{COC}_{6} \mathrm{H}_{4} \mathrm{Br}\right.$, and $o$-disubstituted $\mathrm{C}_{6} \mathrm{H}_{4}$ ), 9.0 (hump, $1 \mathrm{H}, \mathrm{NH} ; \mathrm{D}_{2}$ Oexchange). Anal. Calc. for $\mathrm{C}_{16} \mathrm{H}_{12} \mathrm{BrNO}$. $0.5 \mathrm{H}_{2} \mathrm{O}$ : C, 59.46; 
H, 4.07; N, 4.34. Found: C, 59.49; H, 4.57; N, 4.57\%. MS (FAB+), m/z: $314.08(2.4 \%, \mathrm{M}+\mathrm{H})$, $316.06(2.2 \%, \mathrm{M}+\mathrm{H}+2)$.

2-(4-Chlorobenzoyl)-3-methyl-1H-indole (13a) Yield: $30 \%$, m.p 167-168 ${ }^{\circ}{ }^{1} \mathrm{H}-\mathrm{NMR}$ $\left(\mathrm{CDCl}_{3}, \delta, \mathrm{ppm}\right): 2.3$ (s, 3H, $\mathrm{CH}_{3}$ ), 7.3-8.1 (m, $8 \mathrm{H}, \mathrm{COC}_{6} \mathrm{H}_{4} \mathrm{Cl}$, and $o$-disubstituted $\left.\mathrm{C}_{6} \mathrm{H}_{4}\right), 9.3$ (hump, $1 \mathrm{H}, \mathrm{NH} ; \mathrm{D}_{2}$ Oexchange) as reported. ${ }^{17}$

\section{Preparation of 1-methyl/ethyl-2-benzoyl/ tolyl-3- phenylindoles; $6 c$ and $9 \mathrm{~b}$}

The appropriate N-methyl/ethyl-o-aminophenylketone $(\mathbf{1 b}, \mathbf{c}), 0.02 \mathrm{~mol})$ and the appropriate phenacyl bromide (5a,e; $0.02 \mathrm{~mol}$ ) were treated similarly as under the general method for preparation of 6-13 (a).

2-Benzoyl-1-ethyl-3-phenyl-1H-indole

(6c) Yield: $40 \%$, m.p 82-84 ${ }^{\circ}$ IR $\left(\mathrm{KBr}, \mathrm{cm}^{-1}\right)$ : $1633(\mathrm{C}=\mathrm{O}), \quad 747,693$ (Ar-H). ${ }^{1} \mathrm{H}-\mathrm{NMR}$ $\left(\mathrm{CDCl}_{3}, \delta, \mathrm{ppm}\right): 1.5\left(\mathrm{t}, 3 \mathrm{H}, \mathrm{NCH}_{2} \mathrm{CH}_{3}\right), 4.5$ (q, $\left.2 \mathrm{H}, \mathrm{NCH}_{2} \mathrm{CH}_{3}\right), 7-7.9\left(\mathrm{~m}, 14 \mathrm{H}, \mathrm{C}_{6} \mathrm{H}_{5}, \mathrm{COC}_{6} \mathrm{H}_{5}\right.$, $o$-disubstituted $\left.\mathrm{C}_{6} \mathrm{H}_{4}\right)$. Anal. Calc. for $\mathrm{C}_{23} \mathrm{H}_{19} \mathrm{NO}$ : $\mathrm{C}, 84.89 ; \mathrm{H}, 5.89 ; \mathrm{N}, 4.30$. Found: C, 85.03; H, 6.34; N, 4.34\%.

1-Methyl-2-(4-methylbenzoyl)-3-phenyl1H-indole (9b) Yield: 47\%, m.p 103-104 ${ }^{\circ} .{ }^{1} \mathrm{H}-$ $\mathrm{NMR}\left(\mathrm{CDCl}_{3}, \delta, \mathrm{ppm}\right): 2.3\left(\mathrm{~s}, 3 \mathrm{H}, \mathrm{COC}_{6} \mathrm{H}_{4^{-}}\right.$ $\left.\mathrm{CH}_{3}\right), 4.1\left(\mathrm{~s}, 3 \mathrm{H}, \mathrm{NCH}_{3}\right), 7-8.1\left(\mathrm{~m}, 13 \mathrm{H}, \mathrm{C}_{6} \mathrm{H}_{5}\right.$, $\mathrm{COC}_{6} \mathrm{H}_{4} \mathrm{CH}_{3}, o$-disubstituted $\mathrm{C}_{6} \mathrm{H}_{4}$ ) Anal. Calc. for $\mathrm{C}_{23} \mathrm{H}_{19} \mathrm{NO}$ : $\mathrm{C}, 84.89 ; \mathrm{H}, 5.89 ; \mathrm{N}, 4.30$. Found: C, 84.82; H, 6.32; N, $4.30 \%$.

General procedure for preparation of $\mathrm{N}$ alkyl/aralkyl- 3- phenyl-2-substitutedbenzoylindoles derivatives, (6-9 (b-f)

Powdered sodium hydroxide or potassium hydroxide $(0.025 \mathrm{~mol})$, indole derivative $(\mathbf{6 - 1 3}$ (a); $0.01 \mathrm{~mol})$ and tetrabutylammonium bromide $(0.0025 \mathrm{~mol})$ were mixed and triturated using magnetic stirrer for $10 \mathrm{~min}$ before adding the appropriate alkyl halide $(0.011 \mathrm{~mol})$. Ether $(50 \mathrm{ml})$ was added to the reaction mixture after $6 \mathrm{hrs}$ of trituration. Ethereal solution was then evaporated and the residue was either crystallized from methanol (6b-f, 7b-d, 8b-e and 9b-f) or purified using dry column flash chromatography (7e, $\mathbf{f}$ and $\mathbf{8 f}$ ) where the elution system was hexane: ethyl acetate $(9: 1)$.

2-Benzoyl-1-methyl-3-phenyl-1H-indole

(6b) Yield: 82\%, m.p 87-88 as reported. ${ }^{16}$

2-Benzoyl-1-ethyl-3-phenyl-1H-indole

(6c) Yield: 98\%, m.p 82-84\% It was identical with the sample prepared by the previouslymentioned method.

2-Benzoyl-3-phenyl-1-propyl-1H-indole

(6d) Yield: 85\%, m.p 83-85 $.{ }^{1} \mathrm{H}-\mathrm{NMR}\left(\mathrm{CDCl}_{3}\right.$, $\delta$, ppm): 0.9 (t, $\left.3 \mathrm{H}, \mathrm{NCH}_{2} \mathrm{CH}_{2} \mathrm{CH}_{3}\right), 1.9(\mathrm{~m}, 2 \mathrm{H}$, $\left.\mathrm{NCH}_{2} \mathrm{CH}_{2} \mathrm{CH}_{3}\right), 4.5\left(\mathrm{t}, 2 \mathrm{H}, \mathrm{NCH}_{2} \mathrm{CH}_{2} \mathrm{CH}_{3}\right)$, 7.2-8.2 (m, $14 \mathrm{H}, \mathrm{C}_{6} \mathrm{H}_{5}, \mathrm{COC}_{6} \mathrm{H}_{5}, o$-disubstituted $\mathrm{C}_{6} \mathrm{H}_{4}$ ) Anal. Calc. for $\mathrm{C}_{24} \mathrm{H}_{21} \mathrm{NO}$ : C, 84.92; H, 6.24; N, 4.13. Found: C, 84.84; H, 6.56; N, $4.30 \%$.

2-Benzoyl-1-butyl-3-phenyl-1H-indole

(6e) Yield: $80 \%$, m.p 58-60 ${ }^{\circ} .{ }^{1} \mathrm{H}-\mathrm{NMR}\left(\mathrm{CDCl}_{3}\right.$, $\delta$, ppm): 0.9 (t, 3H, $\mathrm{NCH}_{2} \mathrm{CH}_{2} \mathrm{CH}_{2} \mathrm{CH}_{3}$ ), 1.2-2 $\left(\mathrm{m}, \quad 4 \mathrm{H}, \quad \mathrm{NCH}_{2} \mathrm{CH}_{2} \mathrm{CH}_{2} \mathrm{CH}_{3}\right), \quad 4.6(\mathrm{t}, \quad 2 \mathrm{H}$, $\left.\mathrm{NCH}_{2} \mathrm{CH}_{2} \mathrm{CH}_{2} \mathrm{CH}_{3}\right), 7.2-8.1$ (m, $14 \mathrm{H}, \mathrm{C}_{6} \mathrm{H}_{5}$, $\mathrm{COC}_{6} \mathrm{H}_{5}, o$-disubstituted $\mathrm{C}_{6} \mathrm{H}_{4}$ ). Anal. Calc. for $\mathrm{C}_{25} \mathrm{H}_{23} \mathrm{NO}$ : C, 84.95; H, 6.56; N, 3.96. Found: C, 84.86; H, 7.35; N, 4.11\%.

2-Benzoyl-1-benzyl-3-phenyl-1H-indole

(6f) Yield: $95 \%$, m.p 119-120 $.{ }^{1} \mathrm{H}-\mathrm{NMR}$ $\left(\mathrm{CDCl}_{3}, \delta\right.$, ppm): $5.9\left(\mathrm{~s}, 2 \mathrm{H}, \mathrm{CH}_{2} \mathrm{C}_{6} \mathrm{H}_{5}\right), 7.2-8.1$ (m, $19 \mathrm{H}, \mathrm{C}_{6} \mathrm{H}_{5}, \mathrm{COC}_{6} \mathrm{H}_{5}, o$-disubstituted $\mathrm{C}_{6} \mathrm{H}_{4}$, $\mathrm{CH}_{2} \mathrm{C}_{6} \mathrm{H}_{5}$ ). Anal. Calc. for $\mathrm{C}_{28} \mathrm{H}_{21} \mathrm{NO}$ : C, 86.71; H, 6.46; N, 3.61. Found: C, 86.14; H, 5.44; N, $3.63 \%$.

2-(4-Bromobenzoyl)-1-methyl-3-phenyl1H-indole (7b) Yield: $87 \%$, m.p 133-135 ${ }^{\circ} .{ }^{1} \mathrm{H}-$ $\operatorname{NMR}\left(\mathrm{CDCl}_{3}, \delta, \mathrm{ppm}\right): 4.1$ (s, $\left.3 \mathrm{H}, \mathrm{NCH}_{3}\right), 7.3-$ $8.1\left(\mathrm{~m}, 13 \mathrm{H}, \mathrm{C}_{6} \mathrm{H}_{5}, \mathrm{COC}_{6} \mathrm{H}_{4} \mathrm{Br}, o\right.$-disubstituted $\mathrm{C}_{6} \mathrm{H}_{4}$ ). Anal. Calc. for $\mathrm{C}_{22} \mathrm{H}_{16} \mathrm{BrNO}$ : C, 67.71; H, 4.13; N, 3.59. Found: C, 67.38; H, 4.34; N, $3.55 \%$.

2-(4-Bromobenzoyl)-1-ethyl-3-phenyl-1Hindole (7c) Yield: 70\%, m.p 108-110 . IR $\left(\mathrm{KBr}, \mathrm{cm}^{-1}\right): 1631(\mathrm{C}=\mathrm{O}), 739,696(\mathrm{Ar}-\mathrm{H}) .{ }^{1} \mathrm{H}-$ NMR $\left(\mathrm{CDCl}_{3}, \delta, \mathrm{ppm}\right): 1.5$ (t, $\left.3 \mathrm{H}, \mathrm{NCH}_{2} \mathrm{CH}_{3}\right)$, 4.6 (q, $\left.2 \mathrm{H}, \mathrm{NCH}_{2} \mathrm{CH}_{3}\right), 7.3-8.1\left(\mathrm{~m}, 13 \mathrm{H}, \mathrm{C}_{6} \mathrm{H}_{5}\right.$, $\mathrm{COC}_{6} \mathrm{H}_{4} \mathrm{Br}, o$-disubstituted $\left.\mathrm{C}_{6} \mathrm{H}_{4}\right)$. Anal. Calc. for $\mathrm{C}_{23} \mathrm{H}_{18} \mathrm{BrNO}$ : C, 68.26; $\mathrm{H}, 4.49 ; \mathrm{N}, 3.46$. Found: C, 67.69; H, 4.46; N, 3.27\%.

2-(4-Bromobenzoyl)-3-phenyl-1-propyl-

1H-indole (7d) Yield: 66\%, m.p 141-143 ${ }^{\circ} .{ }^{1} \mathrm{H}-$ $\mathrm{NMR}\left(\mathrm{CDCl}_{3}, \delta, \mathrm{ppm}\right): 0.9$ (t, $3 \mathrm{H}, \mathrm{NCH}_{2} \mathrm{CH}_{2}-$ $\left.\mathrm{CH}_{3}\right), 1.8\left(\mathrm{~m}, 2 \mathrm{H}, \mathrm{NCH}_{2} \mathrm{CH}_{2} \mathrm{CH}_{3}\right), 4.5(\mathrm{t}, 2 \mathrm{H}$, $\left.\mathrm{NCH}_{2} \mathrm{CH}_{2} \mathrm{CH}_{3}\right), 7.2-8.1\left(\mathrm{~m}, 13 \mathrm{H}, \mathrm{C}_{6} \mathrm{H}_{5}, \mathrm{CO}-\right.$ $\mathrm{C}_{6} \mathrm{H}_{4} \mathrm{Br}, o$-disubstituted $\left.\mathrm{C}_{6} \mathrm{H}_{4}\right)$. Anal. Calc. for $\mathrm{C}_{24} \mathrm{H}_{20} \mathrm{BrNO} .0 .5 \mathrm{H}_{2} \mathrm{O}$ : C, 67.39; H, 4.96; N, 3.34. Found: C, 67.64; H, 5.05; N, 3.24\%.

2-(4-Bromobenzoyl)-1-butyl-3-phenyl-1Hindole (7e) Yield: $45 \%$, oil. ${ }^{1} \mathrm{H}-\mathrm{NMR}\left(\mathrm{CDCl}_{3}\right.$, $\delta$, ppm): 0.9 (t, $3 \mathrm{H}, \mathrm{NCH}_{2} \mathrm{CH}_{2} \mathrm{CH}_{2} \mathrm{CH}_{3}$ ), 1.1-2.1 (m, $\left.4 \mathrm{H}, \mathrm{NCH}_{2} \mathrm{CH}_{2} \mathrm{CH}_{2} \mathrm{CH}_{3}\right), 4.5$ (t, $2 \mathrm{H}, \mathrm{NCH}_{2}-$ $\left.\mathrm{CH}_{2} \mathrm{CH}_{2} \mathrm{CH}_{3}\right), 7.2-8.1\left(\mathrm{~m}, 13 \mathrm{H}, \mathrm{C}_{6} \mathrm{H}_{5}, \mathrm{COC}_{6} \mathrm{H}_{4-}\right.$ 
$\mathrm{Br}, o$-disubstituted $\mathrm{C}_{6} \mathrm{H}_{4}$ ). MS (FAB+), m/z: 431.31(2.2\%, M), $433.19(1.7 \%, \mathrm{M}+2)$.

1-Benzyl-2-(4-bromobenzoyl)-3-phenyl1H-indole (7f) Yield: $55 \%$, oil. ${ }^{1} \mathrm{H}-\mathrm{NMR}$ $\left(\mathrm{CDCl}_{3}, \delta, \mathrm{ppm}\right): 5.8\left(\mathrm{~s}, 2 \mathrm{H}, \mathrm{CH}_{2} \mathrm{C}_{6} \mathrm{H}_{5}\right), 7.2-8.1$ $\left(\mathrm{m}, \quad 18 \mathrm{H}, \quad \mathrm{C}_{6} \mathrm{H}_{5}, \quad \mathrm{COC}_{6} \mathrm{H}_{4} \mathrm{Br}, o\right.$-disubstituted $\left.\mathrm{C}_{6} \mathrm{H}_{4}, \mathrm{CH}_{2} \mathrm{C}_{6} \mathrm{H}_{5}\right)$.

2-(4-Chlorobenzoyl)-1-methyl-3-phenyl-

1H-indole (8b) Yield: 85\%, m.p 118-20 ${ }^{\circ}{ }^{1} \mathrm{H}-$ NMR $\left(\mathrm{CDCl}_{3}, \delta, \mathrm{ppm}\right): 4.1$ (s, $\left.3 \mathrm{H}, \mathrm{NCH}_{3}\right)$, 7.2$8.1\left(\mathrm{~m}, 13 \mathrm{H}, \mathrm{C}_{6} \mathrm{H}_{5}, \mathrm{COC}_{6} \mathrm{H}_{4} \mathrm{Cl}, o\right.$-disubstituted $\mathrm{C}_{6} \mathrm{H}_{4}$ ). Anal. Calc. for $\mathrm{C}_{22} \mathrm{H}_{16} \mathrm{ClNO}$ : C, 76.41; H, 4.66; N, 4.05. Found: C, 76.07; H, 4.38; N, $4.04 \%$.

2-(4-Chlorobenzoyl)-1-ethyl-3-phenyl-1Hindole (8c) Yield: 72\%, m.p 58-60 ${ }^{\circ}{ }^{1} \mathrm{H}-\mathrm{NMR}$ $\left(\mathrm{CDCl}_{3}, \delta, \mathrm{ppm}\right): 1.5$ (t, 3H, $\left.\mathrm{NCH}_{2} \mathrm{CH}_{3}\right), 4.6$ (q, $\left.2 \mathrm{H}, \mathrm{NCH}_{2} \mathrm{CH}_{3}\right), 7.2-8.1\left(\mathrm{~m}, 13 \mathrm{H}, \mathrm{C}_{6} \mathrm{H}_{5}\right.$, CO$\mathrm{C}_{6} \mathrm{H}_{4} \mathrm{Cl}, o$-disubstituted $\left.\mathrm{C}_{6} \mathrm{H}_{4}\right)$. Anal. Calc. for $\mathrm{C}_{23} \mathrm{H}_{18}$ CINO: C, 76.77; H, 5.64; N, 3.89. Found: C, 76.45; H, 6.38; N, 3.71\%.

2-(4-Chlorobenzoyl)-3-phenyl-1-propyl$1 \mathrm{H}$-indole (8d) Yield: 68\%, m.p 135-137 $.{ }^{1} \mathrm{H}-$ $\mathrm{NMR}\left(\mathrm{CDCl}_{3}, \delta, \mathrm{ppm}\right): 0.9\left(\mathrm{t}, 3 \mathrm{H}, \mathrm{NCH}_{2}-\right.$ $\left.\mathrm{CH}_{2} \mathrm{CH}_{3}\right), 1.9\left(\mathrm{~m}, 2 \mathrm{H}, \mathrm{NCH}_{2} \mathrm{CH}_{2} \mathrm{CH}_{3}\right), 4.5(\mathrm{t}$, $\left.2 \mathrm{H}, \mathrm{NCH}_{2} \mathrm{CH}_{2} \mathrm{CH}_{3}\right), 7.2-8.2\left(\mathrm{~m}, 14 \mathrm{H}, \mathrm{C}_{6} \mathrm{H}_{5}\right.$, $\mathrm{COC}_{6} \mathrm{H}_{5}, o$-disubstituted $\mathrm{C}_{6} \mathrm{H}_{4}$ ) Anal. Calc. for $\mathrm{C}_{24} \mathrm{H}_{20}$ CINO: C, 77.10; H, 5.39; N, 3.75. Found: C, 76.59; H, 5.68; N, 3.73\%.

1-Butyl-2-(4-chlorobenzoyl)-3-phenyl-1Hindole (8e) Yield: 44\%, m.p 55-56 ${ }^{\circ}{ }^{1} \mathrm{H}-\mathrm{NMR}$ $\left(\mathrm{CDCl}_{3}, \quad \delta, \quad \mathrm{ppm}\right): 0.9 \quad\left(\mathrm{t}, \quad 3 \mathrm{H}, \quad \mathrm{NCH}_{2} \mathrm{CH}_{2-}\right.$ $\left.\mathrm{CH}_{2} \mathrm{CH}_{3}\right), 1.9$ (m, 4H, NCH$\left.{ }_{2} \underline{\mathrm{CH}}_{2} \mathrm{C}_{2} \mathrm{CH}_{3}\right), 4.6$ (t, $\left.2 \mathrm{H}, \mathrm{NCH}_{2} \mathrm{CH}_{2} \mathrm{CH}_{2} \mathrm{CH}_{3}\right), 7.2-8.1(\mathrm{~m}, 13 \mathrm{H}$, $\mathrm{C}_{6} \mathrm{H}_{5}, \mathrm{COC}_{6} \mathrm{H}_{4} \mathrm{Cl}$, o-disubstituted $\left.\mathrm{C}_{6} \mathrm{H}_{4}\right)$. Anal. Calc. for $\mathrm{C}_{25} \mathrm{H}_{22} \mathrm{ClNO}$ : C, 77.41; H, 5.72; N, 3.61. Found: C, 77.02; H, 5.70; N, 3.77\%.

1-Benzyl-2-(4-chlorobenzoyl)-3-phenyl-

1H-indole (8f) Yield: 39\%, oil. ${ }^{1} \mathrm{H}-\mathrm{NMR}$ $\left(\mathrm{CDCl}_{3}, \delta\right.$, ppm): 5.9 (s, $\left.2 \mathrm{H}, \mathrm{CH}_{2} \mathrm{C}_{6} \mathrm{H}_{5}\right), 7.1-8.1$ $\left(\mathrm{m}, \quad 18 \mathrm{H}, \quad \mathrm{C}_{6} \mathrm{H}_{5}, \quad \mathrm{COC}_{6} \mathrm{H}_{4} \mathrm{Cl}, \quad o\right.$-disubstituted $\left.\mathrm{C}_{6} \mathrm{H}_{4}, \mathrm{CH}_{2} \mathrm{C}_{6} \mathrm{H}_{5}\right)$.

1-Methyl-2-(4-methylbenzoyl)-3-phenyl-

1H-indole (9b) Yield: 95\%, m.p 103-104 ${ }^{\circ}$ It is to be noted down that m.p as well as spectral and elemental analyses were all identical with the sample prepared by the previouslymentioned method.

1-Ethyl-2-(4-methylbenzoyl)-3-phenyl-1Hindole (9c) Yield: 90\%, m.p 75-76 . IR (KBr, $\left.\mathrm{cm}^{-1}\right)$ : $1632(\mathrm{C}=\mathrm{O}), 741,697$ (Ar-H). ${ }^{1} \mathrm{H}-\mathrm{NMR}$ $\left(\mathrm{CDCl}_{3}, \delta, \mathrm{ppm}\right): 1.5$ (t, 3H, $\left.\mathrm{NCH}_{2} \mathrm{CH}_{3}\right), 2.3$ (s, $\left.3 \mathrm{H}, \mathrm{COC}_{6} \mathrm{H}_{4} \mathrm{CH}_{3}\right), 4.6$ (q, 2H, $\mathrm{NCH}_{2} \mathrm{CH}_{3}$ ), 7$8.1\left(\mathrm{~m}, 13 \mathrm{H}, \mathrm{C}_{6} \mathrm{H}_{5}, \mathrm{COC}_{6} \mathrm{H}_{4} \mathrm{CH}_{3}\right.$, o-disubstituted $\left.\mathrm{C}_{6} \mathrm{H}_{4}\right)$. Anal. Calc. for $\mathrm{C}_{24} \mathrm{H}_{21} \mathrm{NO}$ : C, 84.92; $\mathrm{H}, 6.24$; N, 4.13. Found: C, 85.22; H, 6.34; N, $4.13 \%$.

2-(4-Methylbenzoyl)-3-phenyl-1-propyl-

1H-indole (9d) Yield: 90\%, m.p 110-112 ${ }^{\circ} .{ }^{1} \mathrm{H}-$ NMR $\left(\mathrm{CDCl}_{3}, \delta, \mathrm{ppm}\right): 0.9\left(\mathrm{t}, 3 \mathrm{H}, \mathrm{NCH}_{2^{-}}\right.$ $\mathrm{CH}_{2} \mathrm{CH}_{3}$ ), 1.9 (m, 2H, $\left.\mathrm{NCH}_{2} \mathrm{CH}_{2} \mathrm{CH}_{3}\right), 2.3$ (s, $\left.3 \mathrm{H}, \mathrm{COC}_{6} \mathrm{H}_{4} \mathrm{CH}_{3}\right), 4.5\left(\mathrm{t}, 2 \mathrm{H}, \mathrm{NCH}_{2} \mathrm{CH}_{2} \mathrm{CH}_{3}\right.$ ), 7-8.1 $\left(\mathrm{m}, 13 \mathrm{H}, \mathrm{C}_{6} \mathrm{H}_{5}, \mathrm{COC}_{6} \mathrm{H}_{4} \mathrm{CH}_{3}, o\right.$-disubstituted $\left.\mathrm{C}_{6} \mathrm{H}_{4}\right)$. Anal. Calc. for $\mathrm{C}_{25} \mathrm{H}_{23} \mathrm{NO}$ : $\mathrm{C}$, 84.95; H, 6.56; N, 3.96. Found: C, 84.91; H, $6.89 ; \mathrm{N}, 4.29 \%$.

1-Butyl-2-(4-methylbenzoyl)-3-phenyl1H-indole (9e) Yield: 65\%, m.p 110-112 ${ }^{\circ}{ }^{1} \mathrm{H}-$ $\mathrm{NMR}\left(\mathrm{CDCl}_{3}, \delta, \mathrm{ppm}\right): 0.9\left(\mathrm{t}, 3 \mathrm{H}, \mathrm{NCH}_{2-}\right.$ $\left.\mathrm{CH}_{2} \mathrm{CH}_{2} \mathrm{CH}_{3}\right), \quad 1-2.2 \quad\left(\mathrm{~m}, \quad 4 \mathrm{H}, \quad \mathrm{NCH}_{2} \mathrm{CH}_{2}-\right.$ $\mathrm{CH}_{2} \mathrm{CH}_{3}$ ), 2.3 (s, $\left.3 \mathrm{H}, \mathrm{COC}_{6} \mathrm{H}_{4} \mathrm{CH}_{3}\right), 4.5$ (t, $2 \mathrm{H}$, $\left.\mathrm{NCH}_{2} \mathrm{CH}_{2} \mathrm{CH}_{3}\right), 7-8.1 \quad\left(\mathrm{~m}, 13 \mathrm{H}, \mathrm{C}_{6} \mathrm{H}_{5}, \mathrm{CO}-\right.$ $\mathrm{C}_{6} \mathrm{H}_{4} \mathrm{CH}_{3}, o$-disubstituted $\mathrm{C}_{6} \mathrm{H}_{4}$ ). Anal. Calc. for $\mathrm{C}_{26} \mathrm{H}_{25} \mathrm{NO}: \mathrm{C}, 84.98 ; \mathrm{H}, 6.86 ; \mathrm{N}, 3.81$. Found: C, 84.86; H, 7.41; N, 4.14\%.

1-Benzyl-2-(4-methylbenzoyl)-3-phenyl$1 \mathrm{H}$-indole (9f) Yield: 55\%, m.p 92-93 ${ }^{\circ}{ }^{1} \mathrm{H}$ NMR $\left(\mathrm{CDCl}_{3}, \delta, \mathrm{ppm}\right): 2.3(\mathrm{~s}, 3 \mathrm{H}, \mathrm{CO}-$ $\left.\mathrm{C}_{6} \mathrm{H}_{4} \mathrm{CH}_{3}\right), 5.8\left(\mathrm{~s}, 2 \mathrm{H}, \mathrm{CH}_{2} \mathrm{C}_{6} \mathrm{H}_{5}\right), 7-8(\mathrm{~m}, 18 \mathrm{H}$, $\mathrm{C}_{6} \mathrm{H}_{5}, \quad \mathrm{COC}_{6} \mathrm{H}_{4} \mathrm{CH}_{3}, \quad o$-disubstituted $\mathrm{C}_{6} \mathrm{H}_{4}$, $\mathrm{CH}_{2} \mathrm{C}_{6} \mathrm{H}_{5}$ ). Anal. Calc. for $\mathrm{C}_{29} \mathrm{H}_{23} \mathrm{NO}$ : C, 86.67; $\mathrm{H}, 5.77$; N, 3.49. Found: C, 86.14; H, 6.36; N, $3.38 \%$.

\section{Synthesis of 1,2-Dibenzoyl-3-phenyl-1H- indole $(6 \mathrm{~g})$}

To a suspension of sodium hydride $(60 \%$, $0.012 \mathrm{~mol}, 0.48 \mathrm{~g})$ in anhydrous DMF $(20 \mathrm{ml})$ was added dropwise a solution of 2-benzoyl-3phenyl-1H-indole $(\mathbf{6 a}, 0.01 \mathrm{~mol}, 2.97 \mathrm{~g})$ in DMF (20 ml), keeping the temperature below $25^{\circ}$. When the addition was complete, the reaction was heated to $40^{\circ}$ until hydrogen evolution ceased. After cooling to $25^{\circ}$, a solution of benzoyl chloride $(0.01 \mathrm{~mol}, 1.16$ $\mathrm{ml})$ in DMF $(10 \mathrm{ml})$ was added dropwise, keeping the temperature below $25^{\circ}$. The mixture was stirred for $2 \mathrm{hrs}$ and then poured onto ice-water $(100 \mathrm{ml})$. The resulting solid was filtered and crystallized from methanol. Yield: $88 \%$, m.p 206-208 . IR $\left(\mathrm{KBr}, \mathrm{cm}^{-1}\right)$ : 1697, 1629 ( 2 bands, $\mathrm{C}=\mathrm{O}$ ), 748, 723, 690 (Ar$\mathrm{H}) .{ }^{1} \mathrm{H}-\mathrm{NMR}\left(\mathrm{CDCl}_{3}, \delta, \mathrm{ppm}\right): 7.2-8.1(\mathrm{~m}, 19 \mathrm{H}$, $\mathrm{C}_{6} \mathrm{H}_{5}, 1$, 2-di- $\mathrm{COC}_{6} \mathrm{H}_{5}, o$-disubstituted $\mathrm{C}_{6} \mathrm{H}_{4}$ ). Anal. Calc. for $\mathrm{C}_{28} \mathrm{H}_{19} \mathrm{NO}_{2}: \mathrm{C}, 83.69 ; \mathrm{H}, 4.77$; N, 3.49. Found: C, 83.07; H, 5.34; N, 3.49\%. 


\section{Pharmacology}

The newly synthesized indole derivatives (6a-g, 7a-f, 8a-f, 9a-13a) were tested for their analgesic effect using hot-plate test in mice. Compounds $\mathbf{7 b}$ and $\mathbf{8 b}$ were tested for their antipyretic and anti-inflammatory effects using yeast induced hyperthermia and paw edema in rats. Both compounds were also tested for their ulcerogenic activity in mice. All compounds were administered to the animals as a solution in $4 \%$ tween 80 . Pure standard indomethacin (Nile co.) was used as a reference in the same dose level. Male albino mice (30-35 g) and male rats $(120-150 \mathrm{~g})$ were used in the biological studies. They were housed in groups of 5 per cage, fed ad libitum with rodent's chow and allowed free access to drinking water. Data were analyzed using the student's $t$-test and the values were expressed as mean \pm S.E. ( $\mathrm{n}=5$ animals).

\section{Analgesic Activity}

Male albino mice (30-35 g) were placed for testing on a hot-plate apparatus, the surface temperature of which was maintained at $55 \pm$ $0.5^{\circ}$. The reaction time in seconds was taken as the time period from the instant the animal reached the hot plate until the moment the animal licked its hind paw or jumped out within a plexiglass cylinder placed on the hotplate. ${ }^{18}$ Animals were randomly divided into groups of 5 mice each and each animal was used once.. Testing was done after $1 \mathrm{hr}$ of the intrapretoneal injection of tested compounds $(5,10 \mathrm{mg} / \mathrm{kg})$, indomethacin $(5,10 \mathrm{mg} / \mathrm{kg})$ or vehicle (control). Mean licking time \pm S.E. was evaluated for each group and results were represented in Fig.2.

\section{Antipyretic activity}

Male albino rats (120-150 g) were made febrile by subcutaneous injection into the dorsal region of brewer's yeast suspension (1 $\mathrm{ml} / 100 \mathrm{~g}, 20 \%$ ) in normal saline at $37^{\circ}$ following Adams et al method. ${ }^{19}$ Then, animals were fasted, but allowed access to water until the termination of experiments that were performed in a temperature-controlled room. Six hours later, those rats which developed a satisfactory pyrexia (at least $1^{\circ}$ higher than normal body temperature $36.8 \pm 0.21$ ) were randomly divided into groups of 5 animals each and received i.p., respectively, tested compounds $(\mathbf{7 b}, \quad \mathbf{8 b}) \quad(5,10 \quad \mathrm{mg} / \mathrm{kg})$, indomethacin $(5,10 \mathrm{mg} / \mathrm{kg})$ or vehicle (control). The rectal temperature was recorded on a thermometer inserted $4 \mathrm{~cm}$ in the rectum for $45 \mathrm{sec}$ before and 1, 2, 3, 4, and $5 \mathrm{hrs}$ after dosing. Results are represented in Fig. 3, 4.

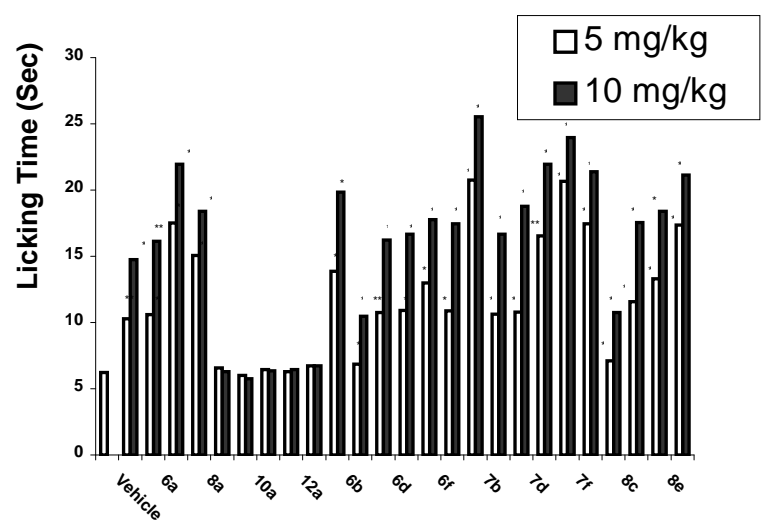

Fig. 2: Mean licking time of 6a-13a, 6b-g, 7bf $\& \mathbf{8 b}-\mathbf{f}$ in the hot-plate test.

Significantly different from control (vehicle) at $* p<0.05, * * p<0.01$

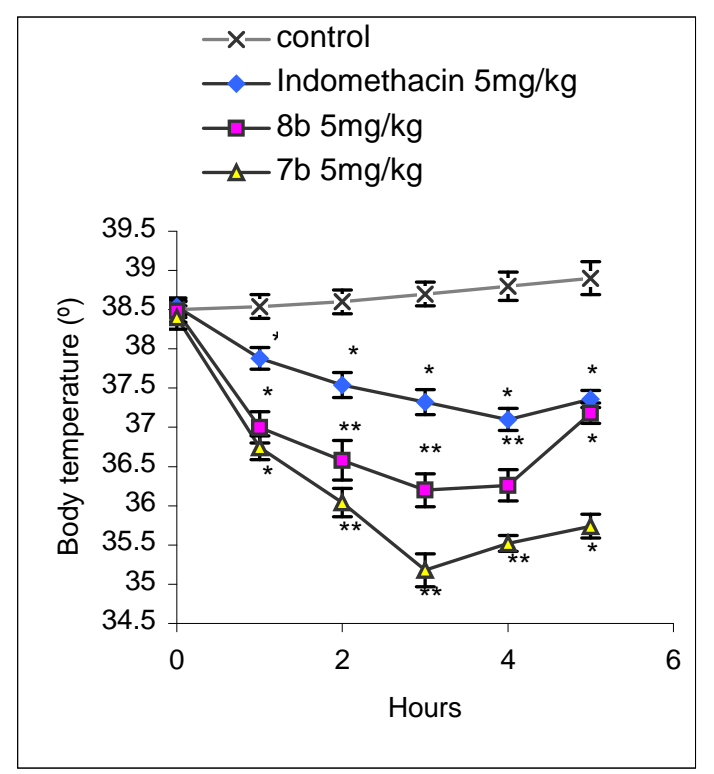

Fig. 3: Antipyretic activity of $\mathbf{7 b} \& \mathbf{8 b}(5 \mathrm{mg} / \mathrm{kg})$ using the yeast induced hyperthermia test. Significantly different from control at $* \mathrm{p}<0.05$, $* * \mathrm{p}<0.01$. 


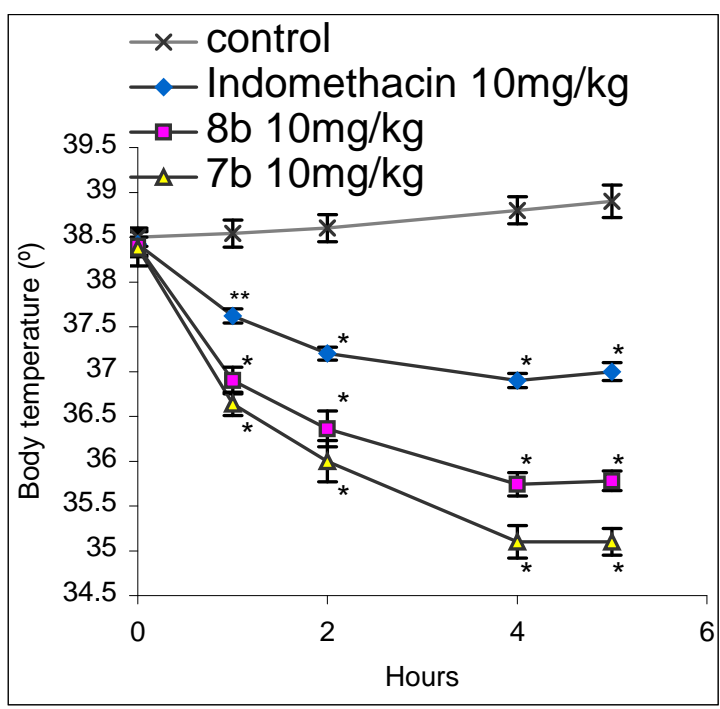

Fig. 4: Antipyretic activity of $7 \mathbf{b} \& \mathbf{8 b}$ (10 $\mathrm{mg} / \mathrm{kg}$ ) using the yeast induced hyperthermia test.

Significantly different from control at $* \mathrm{p}<0.05$, $* * p<0.01$

\section{Anti-inflammatory Activity}

The anti-inflammatory activity was done according to the method described by Winter $e t$ $a l .{ }^{20}$ where a pedal inflammation in rat paws induced by subplantar injection of brewer's yeast suspension $(1 \mathrm{ml} / 100 \mathrm{~g}, 20 \%)$ into the right hind of the rats.
Male albino rats (120-150 g) were randomly divided into groups of 5 animals each. The thickness of rat paw was measured by a Vernier caliper (SMIEC) before and $20 \mathrm{hr}$ after yeast injection to detect the inflammation induced by yeast. Tested compounds $(\mathbf{7 b}$ and 8b) at doses of 5 and $10 \mathrm{mg} / \mathrm{kg}$ were injected i.p. to four different groups of rats. Control group received a vehicle (tween 80 solution), while reference groups received indomethacin i.p. at a dose 5 and $10 \mathrm{mg} / \mathrm{kg}$.

The difference between the thickness of the two paws was taken as a measure of edema. The measurement was carried out at 0.0, 0.5, 1, $2,3,4,5,12$ hrs after injection of the tested compounds, reference and vehicle. The percentage of edema and percentage of inhibition were calculated ${ }^{21}$ and results are listed in Tables 1 and 2.

\section{Gastric ulceration}

Male mice were fasted for $24 \mathrm{hrs}$. Tested compounds were administered orally to groups of 6 mice. After $6 \mathrm{hrs}$, the animals were scarified, the stomachs were removed and gastric lesions on the mucosa were determined by using stereoscopic microscope. 22 "Ulcer" was defined as at least one lesion that was 0.5 $\mathrm{mm}$ or more in length. All lesions of more than $0.1 \mathrm{~mm}$ in length were summed to obtain the ulcer index. Results are listed in Table 3.

Table 1: Effects of compounds $\mathbf{7 b}$ and $\mathbf{8 b}$ on yeast induced paw edema in rats.

\begin{tabular}{|c|c|c|c|c|c|c|c|c|c|}
\hline \multirow{2}{*}{ Group } & \multirow{2}{*}{$\begin{array}{c}\text { Dose } \\
\mathrm{mg} / \mathrm{kg}\end{array}$} & \multicolumn{8}{|c|}{ Percentage of edema (\%) } \\
\hline & & $\mathrm{T}_{0}$ & $0.5 \mathrm{hr}$ & $1 \mathrm{hr}$ & $2 \mathrm{hr}$ & $3 \mathrm{hr}$ & $4 \mathrm{hr}$ & $5 \mathrm{hr}$ & $12 \mathrm{hr}$ \\
\hline Control & -- & 60.97 & 63.31 & 63.57 & 65.47 & 66.01 & 66.16 & 66.96 & 66.96 \\
\hline \multirow{2}{*}{ Indomethacin } & 5 & 55.52 & 53.47 & 52.00 & 52.00 & 46.94 & 47.31 & 48.91 & 59.02 \\
\hline & 10 & 55.20 & 52.40 & 52.40 & 50.87 & 43.59 & 43.59 & 44.61 & 40.50 \\
\hline \multirow[t]{2}{*}{$7 \mathrm{~b}$} & 5 & 57.86 & 52.91 & 49.96 & 47.91 & 41.27 & 42.38 & 42.81 & 58.66 \\
\hline & 10 & 55.63 & 49.80 & 47.56 & 41.08 & 36.41 & 38.21 & 38.96 & 50.29 \\
\hline \multirow[t]{2}{*}{$8 \mathrm{~b}$} & 5 & 57.15 & 52.46 & 50.93 & 49.30 & 43.66 & 44.08 & 45.92 & 59.44 \\
\hline & 10 & 57.26 & 52.92 & 50.64 & 48.48 & 45.55 & 41.60 & 41.82 & 16.84 \\
\hline
\end{tabular}


Table 2: Inhibitory effect of compounds $\mathbf{7 b}$ and $\mathbf{8 b}$ on yeast induced paw edema in rats.

\begin{tabular}{|c|c|c|c|c|c|c|c|c|c|}
\hline \multirow{2}{*}{ Group } & \multirow{2}{*}{$\begin{array}{c}\text { Dose } \\
\mathrm{mg} / \mathrm{kg}\end{array}$} & \multicolumn{7}{|c|}{ Percentage of edema inhibition } \\
\cline { 3 - 10 } & & $\mathrm{T}_{0}$ & $0.5 \mathrm{hr}$ & $1 \mathrm{hr}$ & $2 \mathrm{hr}$ & $3 \mathrm{hr}$ & $4 \mathrm{hr}$ & $5 \mathrm{hr}$ & $12 \mathrm{hr}$ \\
\hline \multirow{2}{*}{ Indomethacin } & 5 & 20.75 & 33.96 & 38.46 & 43.35 & 62.05 & 54.43 & 53.15 & 29.56 \\
\cline { 3 - 10 } & 10 & 21.17 & 36.24 & 36.96 & 45.42 & 60.20 & 60.46 & 60.25 & 66.39 \\
\hline \multirow{2}{*}{$7 \mathrm{~b}$} & 5 & 12.36 & 35.10 & 42.96 & 51.64 & 63.91 & 62.47 & 63.16 & 30.21 \\
\cline { 3 - 10 } & 10 & 12.36 & 35.10 & 42.96 & 51.64 & 63.91 & 62.47 & 63.16 & 30.21 \\
\hline \multirow{2}{*}{$8 \mathrm{~b}$} & 5 & 14.88 & 36.24 & 40.71 & 48.87 & 60.20 & 59.79 & 58.31 & 27.94 \\
\cline { 3 - 9 } & 10 & 14.04 & 34.72 & 41.08 & 50.25 & 56.82 & 63.48 & 64.45 & 89.98 \\
\hline
\end{tabular}

Table 3: Effect of compounds $\mathbf{7 b}$ and $\mathbf{8 b}$ on gastric mucosa of mice.

\begin{tabular}{||c|c|c|c||}
\hline \multirow{3}{*}{ Group } & $\begin{array}{c}\text { Dose } \\
\mathrm{mg} / \mathrm{kg}\end{array}$ & $\begin{array}{c}\text { Ratio of } \\
\text { ulcerated } \\
\text { animals }\end{array}$ & $\begin{array}{c}\text { Ulcer index } \\
\text { (mean } \pm \text { S.E.) }\end{array}$ \\
\hline \multirow{3}{*}{$\begin{array}{c}\text { Indome- } \\
\text { thacin }\end{array}$} & 5 & $2 / 6$ & $0.85 \pm 0.07$ \\
\cline { 2 - 4 } & 10 & $4 / 6$ & $1.76 \pm 0.12$ \\
\cline { 2 - 4 } & 30 & $6 / 6$ & $2.10 \pm 0.17$ \\
\cline { 2 - 4 } $7 \mathrm{~b}$ & 50 & Not tested & -- \\
\hline \multirow{4}{*}{$8 \mathrm{~b}$} & 5 & $0 / 6$ & 0.00 \\
\cline { 2 - 4 } & 10 & $0 / 6$ & 0.00 \\
\cline { 2 - 4 } & 30 & $2 / 6$ & $1.50 \pm 0.07$ \\
\cline { 2 - 4 } & 50 & $4 / 6$ & $1.50 \pm 0.07$ \\
\cline { 2 - 4 } & 5 & $0 / 6$ & 0.00 \\
\cline { 2 - 4 } & 10 & $2 / 6$ & $1.35 \pm 0.07$ \\
\cline { 2 - 4 } & 30 & $3 / 6$ & $1.38 \pm 0.02$ \\
\hline \hline
\end{tabular}

\section{RESULTS AND DISCUSSION}

\section{Chemistry}

Monoalkylation of o-aminoketones; (1b-c, 2b-c)

Polyalkylation is the major drawback limiting the use of direct alkylation of primary amines in synthesis of secondary amines. Different methods were developed to solve this problem. $^{15,23}$ In the present work, it seemed reasonable to affect monoalkylation of $O$ - aminoketones through tosylation of these amines $^{15}$ followed by $N$-alkylation ${ }^{15}$ and hydrolysis of $N$-alkyl- $N$-tosylated derivatives, ${ }^{24}$ Fig. 1.

Preparation of N-alkyl-3-phenyl-2aroylindoles; (6c, 9b)

Cyclisation of the prepared $N$-alkyl-oaminobenzophenones (1), c) with the appropriate phenacyl bromide $(\mathbf{5 a}, \mathbf{b})$ to give 1alkyl-3-phenyl-2-aroylindoles was attempted, Fig. 1. This approach proved to be of limited utility due to formation of non-purifiable resinous products, low overall yield and being a multistepped method. Therefore, another approach was successfully adopted.

\section{Synthesis of 3-methyl/phenyl -2-aroylindoles (6a-13a)}

Compounds (6a, 8a, 9a, 11a and 13a) are reported to be prepared by treating the appropriately substituted o-amino-phenyl ketone with $\alpha$-haloketones. ${ }^{16,17}$ In the present study the same method was adopted to prepare similar compounds (7a, 10a \& 12a) as confirmed by elemental and spectral analyses. EI spectra of compounds $(\mathbf{7 a}, \mathbf{1 2 a})$ didn't show the molecular ion peak. However, fast atomic bombardment (FAB) technique revealed the presence of quasimolecular ion peaks: 7a; $\left(\mathrm{M}^{+\cdot}+\mathrm{H}+2\right)$ at $\mathrm{m} / \mathrm{z} 378.9$ corresponding to $\left(\mathrm{C}_{21} \mathrm{H}_{14}{ }^{81} \mathrm{BrNO}+\mathrm{H}\right)$ and $\left(\mathrm{M}^{+\cdot}+\mathrm{H}\right)$ at $\mathrm{m} / \mathrm{z} 376.9$ 
corresponding to $\left.\left(\mathrm{C}_{21} \mathrm{H}_{14}{ }^{79} \mathrm{BrNO}+\mathrm{H}\right)\right)$ and 12a; $\left(\mathrm{M}^{+\cdot}+\mathrm{H}+2\right)$ at $\mathrm{m} / \mathrm{z} 316$ corresponding to $\left(\mathrm{C}_{16} \mathrm{H}_{12}{ }^{81} \mathrm{BrNO}+\mathrm{H}\right)$ and $\left(\mathrm{M}^{+\cdot}+\mathrm{H}\right)$ at $\mathrm{m} / \mathrm{z} 314$ corresponding to $\left(\mathrm{C}_{16} \mathrm{H}_{12}{ }^{79} \mathrm{BrNO}+\mathrm{H}\right)$.

\section{Synthesis of 1-alkyl-2-aroyl-3-phenylindoles (6b-f, 7b-f, 8b-f, 9b-f)}

$\mathrm{N}$-Alkylation of aroylindoles was successfully achieved adopting solid /liquid phase transfer catalysis SL-PTC ${ }^{25,26}$ using tetrabutylammonium bromide (TBAB) as a catalyst and without solvent. Structural elucidation of the prepared compounds was confirmed by elemental and spectral analyses. Although, the molecular ion peak was absent in EI spectrum of compound $\mathbf{7 e}$, it could be recognized through $\mathrm{FAB}$ spectrum by the presence of ion peaks; $\left(\mathrm{M}^{+*}+2\right)$ at $\mathrm{m} / \mathrm{z} 433$ corresponding to $\left(\mathrm{C}_{25} \mathrm{H}_{22}{ }^{81} \mathrm{BrNO}\right)$ and $\left(\mathrm{M}^{+\bullet}\right)$ at $\mathrm{m} / \mathrm{z} 431$ corresponding to $\left(\mathrm{C}_{25} \mathrm{H}_{22}{ }^{79} \mathrm{BrNO}\right)$. It is to be noted down that PTC seems to be a superior technique in terms of yield, purity of products and non-tediousness of reaction.

\section{Synthesis of 1,2-Dibenzoyl-3-phenylindole (6g)}

$\mathrm{N}$-Acetylation of indoles is reported to proceed using acetic anhydride/ $\mathrm{K}_{2} \mathrm{CO}_{3}{ }^{27,}{ }^{28} \mathrm{In}$ the present work, $\mathrm{N}$-benzoylation of 6 a to yield $6 \mathrm{~g}$ was achieved using sodium hydride and benzoyl chloride.

\section{Pharmacology}

Analgesic activity

The effect of tested compounds or reference drug on mean licking time in hotplate test was used as a measure of analgesic activity. It was compared at 5 and $10 \mathrm{mg} / \mathrm{kg}$ dose levels with a control group which was injected with vehicle. Results presented in Fig.2 revealed that; among the 2-aroyl-3phenylindoles (6a-10a), compounds that showed significant analgesic activity were those (un)substituted by halogen at the para position of 2-aroyl entity (-, $\mathrm{Br}, \mathrm{Cl} ; \mathbf{6 a}, 7 \mathbf{a}, \mathbf{8 a}$ respectively). Compounds substituted by methyl or nitro were found to be inactive in regard to both doses. Also, 2-aroyl-3methylindoles (11a-13a) were devoid of analgesic activity at both doses.

The effect of substitution at nitrogen atom of certain indoles on analgesic activity was examined. Results indicate that N-substitution on the indole ring by groups such as methyl, propyl, butyl, benzyl or benzoyl in compounds $6,7,8(b, d, e, f)$ and $6 \mathrm{~g}$, increases the analgesic activity, with the exception of $\mathrm{N}$ ethyl derivatives; $(\mathbf{6 c}, \mathbf{7 c}$ and $\mathbf{8 c})$. The highest activity was found to reside in $N$-methyl derivatives 7 and $\mathbf{8}$ (b); Fig. 2.

\section{Antipyretic activity}

Investigation of the effects of tested compounds (7b, 8b) compared with indomethacin on yeast induced hyperthermia test, represented in Fig. 3, 4, indicates that they exhibit significantly marked reduction in rectal temperature. Also, animals reached normal temperature after $1 \mathrm{hr}$ in case of compounds $\mathbf{7 b}$ and $\mathbf{8 b}$ and within 3-4 hrs in case of indomethacin

\section{Anti-inflammatory activity}

Effects of tested compounds $(\mathbf{7 b}, \mathbf{8 b})$ and indomethacin on yeast induced inflammation were represented as the time course of edema, (Table 1). At $5 \mathrm{mg} / \mathrm{kg}$-dose level, results indicated that tested compounds and indomethacin caused a marked decrease in edema if compared to control group with slight faster and more effective reduction in case of tested compounds. Also, a decrease in activity was noticeable after $5 \mathrm{hrs}$ of administration of both tested compounds and indomethacin. At $10 \mathrm{mg} / \mathrm{kg}$-dose level, a decrease in the activity of compound (7b) was noticed after 5 hrs while compound $(\mathbf{8 b})$ persisted in reducing edema during 12 hrs period and making an optimum reduction at the end of the period (edema\% $=$ $10 \%$ ). In a similar way, the comparison may utilize the inhibitory effects of tested compounds and indomethacin on the yeast induced edema versus time as represented in Table 2.

\section{Gastric ulceration}

Occurrence of gastrointestinal damage (bleeding, ulceration) is probably the most prevalent and serious side effect associated with the use of NSAIDs. ${ }^{29}$ Observation of the gastrointestinal mucosa for the presence of lesions following oral administration of a single dose of the tested compounds or reference drug has been taken as an indication for the ulcerogenic effects. Both the frequency of ulceration (expressed as ratio of ulcerated 
animals) and the severity of ulceration (expressed as ulcer index) were used for comparison of the tested compounds and indomethacin. Results represented in Table 3 indicated that compound $\mathbf{7 b}$ has no ulcerogenic toxicity at treating doses $(5,10 \mathrm{mg} / \mathrm{kg})$ while compound $\mathbf{8 b}$ caused mild ulceration in one third of the animals at $10 \mathrm{mg} / \mathrm{kg}$. At higher doses both tested compounds exhibited lower ulcerogenic activity compared with indomethacin which caused ulceration at all doses increased in frequency and severity as increasing doses.

\section{REFERENCES}

1- W. C. Black, M. Bayly, C. C. Chan and S. Charleson, Bioorg. Med. Lett., 6, 725 (1996).

2- A. S. Kalgutkar, A. B. Marrett, B. C. Crews and R. P. Remnel, L. J. Marrett, J. Med. Chem., 43, 2860 (2000).

3- K. J. Doebel and J. W. F. Wasley, U.S., 3, 505, 354 (1970), through Chem. Abstr., 73, 14690u (1970).

4- Roussel-Uclaf Fr., 1, 584, 808 (1970), through Chem. Abstr., 74, 141524p (1971).

5- T. E. D`Ambra, E. R. Bacon, M. R. Bell, P. M. Carabateas and M. A. Eissentat, Eur. Pat. Appl., EP444, 451 (1991), through Chem. Abstr., 116, 6406x (1992).

6- W. Huang, Z. Yang, and S. Peng, Yaoxue Xuebao, 20, 10, 737 (1985), through Chem. Abstr., 105, 42591k (1986).

7- M. R. Bell, Eur. Pat. Appl., EP171, 037, (1986), through Chem. Abstr., 105, 78832d (1986).

8- A. E. Branstrom, S. A. I. Ingemar and A. Gagneux, S. Africa, 6800, 509 (1968), through Chem. Abstr., 70, 77778n (1969).

9- A. E. Branstrom, S. A. I. Carlson and A. Gagneux, Ger. Offen., 1, 937, 020 (1970), through Chem. Abstr., 73, 14688z (1970).

10- A. E. Branstrom, S. A. I. Carlson and A. Gagneux, Swiss, 498, 833 (1970), through Chem. Abstr., 74, 141519r (1971).
11- J. R. Carson and S. Wong, J. Med. Chem., 16, 172 (1973).

12- G. Dannhardt, S. Laufer, Curr. Med. Chem., 7, 1101 (2000).

13- G. Dannhardt, L. B. Fiebich and J. Schweppenhauser, Eur. J. Med. Chem., 37, 147 (2002).

14- G. Dannhardt, W. Kiefer, G. Kramer and L. Fiebich, Eur. J. Med. Chem., 35, 499 (2000).

15- J. B. Hendrickson, R. Bergeron, and D. D. Sternbach, Tetrahedron, 31, 2517 (1975).

16- C. D. Jones and T. Suarez, J. Org. Chem., 37, 23, 3622 (1972).

17- Ibid., 37, 23, 3624 (1972).

18- N. B. Eddy and D. Leimbach, J. Pharmacol. Exp. Ther., 139, 107 (1963).

19- S. S. Adams, P. Hebborn, J. S. Nicholson, J. Pharm. Pharmacol., 20, 305 (1968).

20- C. A. Winter, E. A. Risley and G. W. Nuss, Proc. Exp. Biol. Med., 111, 544 (1962).

21- E. Valencia and M. Feria, J. G. Diaz, A. Gonzalez, J. Bermejo, Planta Med., 60, 395 (1994).

22- H. Ikuta, H. Shirota, S. Kobayashi, Y. Yamagishi, K. Yamada, I. Yamatsu and K. Katayama, J. Med. Chem., 30, 1995 (1987).

23- R. M. Roberts, P. J. Vogt and J. Amer. Chem. Soc., 78, 4778 (1965).

24- S. Furniss Brian, J. Hannaford Antony, W. G. Smith Peter, R. Tatchell Austin, "Vogel's Textbook of Practical Organic Chemistry" (Fifth Ed.), Longman, England, 1991, p. 1230.

25- M. Bourak and R. Gallo, Heterocycles, 31, 3 (1990).

26- J. Barry, G. Bram, G. Decodts, A. Loupy, P. Pigeon and J. Sansoulet, Tetrahedron 39, 2669 (1983).

27- H. Takechi, M. Machida, and Y. Kanaoka, Chem. Pharm. Bull., 36, 3770 (1988).

28- D. Dhanak and C. B. Reese, J. Chem. Soc., Perkin Trans., 1, 2181 (1986).

29- Rainsford K. D., Agents and Actions, 7, 573 (1977). 